\title{
Fatal Abdominal Compartment Syndrome After Endoscopic Gastric Bypass Revision
}

\author{
Daniel Swartz, MD \\ Central California Institute of Minimally Invasive Surgery, Fresno, CA, USA.
}

\begin{abstract}
Introduction: This case report describes a procedure-related mortality due to abdominal compartment syndrome following endoscopic stomal reduction as a revisional procedure for Roux-en-Y gastric bypass. This procedure is increasing in popularity due in part to the well-documented safety profile.

Case Description: The patient presented recurrent morbid obesity and GERD 11 years following Roux-en-Y gastric bypass and underwent a laparoscopic hiatal hernia repair and transoral outlet reduction. Severe distension developed following this 45-minute endoscopic procedure leading to ischemia of the bilateral lower extremities. Urgent laparotomy was performed to relieve the compartment syndrome but the patient developed refractory disseminated intravascular coagulation (DIC), cardiogenic shock, and succombed.
\end{abstract}

Discussion: Although a rare event, patients undergoing prolonged endoscopic procedure using air insufflation under general anesthesia may be at risk.

Key Words: Endoluminal stomal reduction, Endoscopic revision, Outlet reduction.

Citation Swartz D. Fatal abdominal compartment syndrome after endoscopic gastric bypass revision. CRSLS e2014.00191. DOI: 10.4293/CRSLS.2014.00191.

Copyright (c) 2015 by SLS, Society of Laparoendoscopic Surgeons. This is an open-access article distributed under the terms of the Creative Commons Attribution-Noncommercial-ShareAlike 3.0 Unported license, which permits unrestricted noncommercial use, distribution, and reproduction in any medium, provided the original author and source are credited.

The author is a consultant for Apollo Endosurgery; however, he has not received any honoraria, consultation fees, or payments of any kind.

Address correspondence to: Daniel Swartz, MD, Central California Institute of Minimally Invasive Surgery, 7060 N Recreation Ave, Ste 101, Fresno, CA 93720, USA. Telephone: (559) 299-9105, Fax: (559) 299-9147, E-mail: deswartzmd@aol.com

\section{INTRODUCTION}

Endoluminal stomal reduction as a revision technique for Roux-en-Y gastric bypass has been reported extensively in the literature. ${ }^{1-8}$ Many devices including the Endocinch (C.R. Bard, Murray Hill, New Jersey); Stomaphyx (Endogastric Solutions, San Mateo, California); Rose procedure (USGI Medical, San Clemente, California); and most recently, Overstitch (Apollo Endosurgery, Austin, Texas) have been shown to be feasible and safe with modest efficacy in the short term. Surgical revision procedures for Roux-en-Y gastric bypass have been associated with a higher rate of complications, including leakage, when compared with primary gastric bypass surgery.9-11 The long-term efficacy of surgical revisions has not been consistently shown, leading to an increase in the popularity of lower-risk endoluminal revisions. All of the endoluminal devices have been shown to have very low morbidity rates and, until recently, no reported deaths. This case report describes a death after endoluminal gastric bypass revision.
Our center began performing endoluminal stomal reduction with or without gastric pouch reduction in 2009 in selected patients using the Rose procedure. Our 15-month follow-up results on the first 18 patients were reported at the 2011 Society of American Gastrointestinal and Endoscopic Surgeons meeting in Orlando, Florida. ${ }^{12}$ Because of technical difficulties requiring rigid anatomic inclusion criteria that limited the use of the Rose procedure in a small minority of patients, we changed to the Overstitch device in October 2012. To date, $>22$ Rose procedures and 78 Overstitch procedures have been performed at our facility without-excluding the described case-any major complications, the need for subsequent surgery, or any deaths.

\section{CASE DESCRIPTION}

The patient was a 55-year-old white female homemaker receiving disability payments because of chronic back 
pain from recurrent morbid obesity after laparoscopic gastric bypass 11 years earlier. Before the gastric bypass procedure, she weighed $216 \mathrm{lb}$ (body mass index [BMI], $36 \mathrm{~kg} / \mathrm{m}^{2}$ ); after the procedure, she achieved a weight of $154 \mathrm{lb}$ (BMI, $26.5 \mathrm{~kg} / \mathrm{m}^{2}$ ) and remained at this weight for 8 years. She then began to regain all of the weight because of a complete loss of restriction/satiety combined with a return to maladaptive eating habits and, because of her chronic back pain, an inability to exercise. At presentation to our office, she weighed $215 \mathrm{lb}$ (BMI, $35.8 \mathrm{~kg} / \mathrm{m}^{2}$ ). In addition, daily heartburn, regurgitation, and chronic cough refractory to proton pump inhibitor therapy had developed. She was offered matriculation into our medical weight loss program to be followed by our physician, nutritionist, fitness trainer, and psychologist, but because she resided 6 hours away, she was provided a set of written guidelines to re-establish appropriate eating and exercise habits. An endoscopic evaluation showed a wide hiatal hernia, a 6-cm-long mildly dilated pouch, and a $5-\mathrm{cm}$ dilated gastrojejunostomy. The patient was offered a laparoscopic hiatal hernia repair with either surgical revision or endoluminal reduction of the stoma and, possibly, the pouch. After a discussion of the risks and benefits, she opted and consented to undergo the latter. Preparation for surgery included a clear liquid diet for 24 hours and $250 \mathrm{~mL}$ of magnesium citrate before surgery.

At the time of surgery, the patient received parenteral prophylactic antibiotics (cefazolin, $2 \mathrm{~g}$ ). She underwent a laparoscopic hiatal hernia repair with posterior cruroplasty with 2 interrupted figure-of-8 Gore-Tex sutures (W. L. Gore \& Associates, Flagstaff, Arizona), and intraoperative endoscopy was performed to confirm a favorable anatomy for endoluminal reduction per our standard technique. The laparoscopic ports were removed, the incisions were closed, and endoluminal outlet reduction was performed using a technique previously described in the medical literature ${ }^{4}$ : The mucosa was endoscopically ablated with bipolar cautery, and a series of 4 No. 2-0 Prolene sutures (Ethicon, Somerville, New Jersey) were placed using a short, running technique with full-thickness bites beginning from right to left. The last suture was cinched over a pneumatic balloon inflated to $7 \mathrm{~mm}$, also per our standard technique. No pouch reduction was performed, and the insufflated air was removed from the upper gastrointestinal tract as much as possible. The entire procedure took 90 minutes, of which 45 minutes was used for the endoscopic component.

The patient showed marked distention at the conclusion of surgery but not uncommonly so, and in the normal course of recovery, these patients tend to pass flatus on recovery from general anesthesia when the abdominal muscles begin to contract. However, this patient continued to have distention and moderate hypotension and did not pass flatus. In the postanesthesia care unit, she was noted to have mottling of her lower extremities with absent pedal pulses. With the patient unable to pass flatus and with progressive hypotension developing, an initial attempt to perform colonoscopic decompression was unsuccessful and the patient was brought back to the operating room for urgent laparotomy. This was performed, and once the tensely distended air-filled bowel was released from the abdominal compartment, her legs became warm and all signs of ischemia resolved. There was no evidence of abdominal ischemia, and urine output was maintained. The bowel was completely decompressed through 1 enterotomy and 1 colotomy, and without any tension or edema in the abdominal wall or viscera, the laparotomy wound was closed without tension while we performed monitoring for any signs of ischemia. The patient remained intubated and was referred to the intensive care unit requiring a small amount of pressor therapy. However, within 1 hour in the intensive care unit, she had severe hypotension not responding to maximal pressor therapy, and profound disseminated intravascular coagulation (DIC) developed, with an international normalized ratio of 22 , prothrombin time of 136 , partial thromboplastin time of $>120$, fibrinogen concentration of $<60 \mathrm{mg} / \mathrm{mL}$, platelet count of 76000 , and D-dimer level of $>10000 \mathrm{ng} / \mathrm{mL}$. Despite the administration of extensive fresh-frozen plasma, cryoprecipitate, and desmopressin acetate, oozing from the midline incision developed, and in the course of several hours, she progressed to unresponsive cardiogenic shock and asystolic cardiac arrest not amenable to resuscitative measures and died shortly thereafter. The cause of death was believed to be abdominal compartment syndrome due to massive small and large bowel distention leading to ischemia- and reperfusion-induced systemic inflammatory response syndrome with profound DIC and cardiogenic shock ultimately leading to cardiac arrest. There was no evidence of intra-abdominal bleeding or signs of peritonitis or sepsis. The endoscopically placed sutures were intact, and no sign of leakage was seen. There was no evidence of abdominal organ ischemia. An incidental finding of sarcoidosis in the lungs was believed to be noncontributory. Although DIC was present, no etiology for this was found on autopsy.

\section{DISCUSSION}

This case represents a procedure-related death after endoscopic outlet reduction following gastric bypass with laparoscopic hiatal hernia repair. The cause of 
post-endoscopy abdominal compartment syndrome is unclear because lengthy endoscopic procedures with patients under general anesthesia, such as endoscopic retrograde cholangiopancreatography and balloon enteroscopy, are commonly performed using air insufflation. Changing to carbon dioxide insufflation might reduce the likelihood of such an event. After this tragic outcome occurred, a thorough re-evaluation of the procedure performed led to 2 changes. First, a carbon dioxide insufflator is now used during all endoluminal surgical procedures, and second, it is now routine for an assistant to hold a laparoscopic bowel clamp for the entire endoluminal procedure. However, it is beyond the scope of this case review to make any formal recommendations because this is the first published report of a post-procedure death. In addition, we carefully monitor patients for signs of lower extremity and abdominal organ ischemia and for systemic hypotension because these might be early warning signs of abdominal compartment syndrome with vascular compromise.

Although the abdominal compartment syndrome was unquestionably due to the endoscopic insufflation of the small and large bowel, causes of DIC other than a reperfusion event merit consideration. It is possible that another etiology such as a drug reaction could have occurred. The medications that the patient received during surgery were levofloxacin, metronidazole, fentanyl, propofol, rocuronium, ondansetron, metoclopramide, ephedrine, and phenylephrine. None of these are associated with the adverse effect of DIC. Other causes of DIC including sepsis, trauma, pancreatitis, malignancy, transfusion reactions, vascular abnormalities, hepatic failure, transplant rejection, heat stroke, and hyperthermia did not occur. A postmortem examination was performed, and no cause of death or any findings to suggest the suspected clinical cause could be found.

\section{References:}

1. Mikami D, Needleman B, Narula V, Durant J, Melvin WS. Natural orifice surgery: initial US experience utilizing the StomaphyX device to reduce gastric pouches after Roux-en-Y gastric bypass. Surg Endosc. 2010;24:223-228.

2. Herron DM, Birkett DH, Thompson CC, Bessler M, Swanström LL. Gastric bypass pouch and stoma reduction using a transoral endoscopic anchor placement system: a feasibility study. Surg Endosc. 2008;22:1093-1099.

3. Kumar N, Thompson CC. Comparison of a superficial suturing device with a full-thickness suturing device for transoral outlet reduction (with videos). Gastrointest Endosc. 2014;79: 984-989.

4. Thompson CC, Chand B, Chen YK, et al. Endoscopic suturing for transoral outlet reduction increases weight loss after Roux-en-Y gastric bypass surgery. Gastroenterology. 2013;145: $129-137$.

5. Jirapinyo P, Slattery J, Ryan MB, Abu Dayyeh BK, Lautz DB, Thompson CC. Evaluation of an endoscopic suturing device for transoral outlet reduction in patients with weight regain following Roux-en-Y gastric bypass. Endoscopy. 2013;45:532-536.

6. Abu Dayyeh BK, Jirapinyo P, Weitzner Z, et al. Endoscopic sclerotherapy for the treatment of weight regain after Roux-en-Y gastric bypass: outcomes, complications, and predictors of response in 575 procedures. Gastrointest Endosc. 2012;76:275282.

7. Brethauer SA, Chand B, Schauer PR, Thompson CC. Transoral gastric volume reduction as intervention for weight management: 12-month follow-up of TRIM trial. Surg Obes Relat Dis. 2012;8:296-303.

8. Horgan S, Jacobsen G, Weiss GD, et al. Incisionless revision of post-Roux-en-Y bypass stomal and pouch dilation: multicenter registry results. Surg Obes Relat Dis. 2010;6:290-295.

9. Park JY, Song D, Kim YJ. Causes and outcomes of revisional bariatric surgery: initial experience at a single center. Ann Surg Treat Res. 2014;86:295-301.

10. Brethauer SA, Kothari S, Sudan R, et al. Systematic review on reoperative bariatric surgery: American Society for Metabolic and Bariatric Surgery Revision Task Force. Surg Obes Relat Dis. 2014; 10(5):952-972.

11. McKenna D, Selzer D, Burchett M, Choi J, Mattar SG. Revisional bariatric surgery is more effective for improving obesityrelated co-morbidities than it is for reinducing major weight loss. Surg Obes Relat Dis. 2014;10:654-659.

12. Swartz D, Felix EL. Endoscopic revision of Roux-en-Y gastric bypass: Effect on recidivist weight gain, gastroesophageal reflux disease and chronic dumping syndrome. Presented at: 28th Annual Meeting of the American Society of Metabolic and Bariatric Surgery; June 2011; Orlando, FL. 